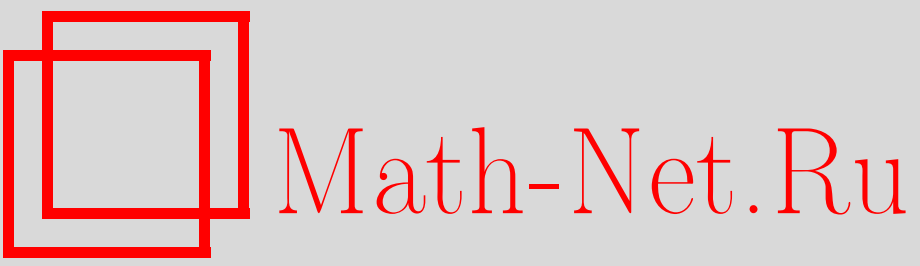

В. Г. Горбунов, А. П. Исаев, О. В. Огиевецкий, БРСТ-оператор для квантовых алгебр Ли: связь с bar-комплексом, ТМФ, 2004, том 139, номер 1, 29-44

DOI: https://doi.org/10.4213/tmf40

Использование Общероссийского математического портала Math-Net.Ru подразумевает, что вы прочитали и согласны с пользовательским соглашением

http://www.mathnet.ru/rus/agreement

Параметры загрузки:

IP: 54.174 .149 .18

26 апреля 2023 г., 15:33:44 


\author{
ТЕОРЕТИЧЕСКАЯ \\ И МАТЕМАТИЧЕСКАЯ \\ ФИЗИКА \\ Том 139, № 1 \\ апрель, 2004
}

(ㄷ) 2004 г. $\quad$ В.Г. Горбунов* , А.П. Исаев ${ }^{\dagger}$, О.В. Огиевецкий ${ }^{\ddagger}$

\title{
БРСТ-ОПЕРАТОР ДЛЯ КВАНТОВЫХ АЛГЕБР ЛИ: СВЯЗЬ С ВАR-КОМПЛЕКСОМ
}

Квантовые алгебры Ли обобщают (супер)алгебры Ли и представляют собой важный класс квадратичных алгебр, возникающих в исчислении Вороновича на квантовых группах. Многие понятия теории (супер)алгебр Ли обобщаются на "квантовый" случай. В частности, имеется БРСТ-оператор $Q\left(Q^{2}=0\right)$, который порождает диф̆ференциал в теории Вороновича и дает информацию о (ко)гомологиях квантовых алгебр Ли. В наших предыдущих работах было сформулировано и решено рекуррентное соотношение для оператора $Q$ квантовых алгебр Ли. В данной работе рассматриваются bar-комплекс для $q$-алгебр Ли и его подкомплекс $q$-антисимметричных цепей. Устанавливается цепное отображение (являющееся изоморфизмом) стандартного комплекса для $q$-алгебры Ли в подкомплекс антисимметричных цепей. Для этого используется ряд нетривиальных тождеств в групповой алгебре группы кос. Обсуждается также обобщение стандартного комплекса на случай, когда $q$-алгебра Ли снабжена оператором градуировки.

Ключевые слова: БРСТ-оператор, квадратичные алгебры, квантовые алгебры Ли, bar-комплекс.

\section{1. ВВЕДЕНИЕ}

Исчисление Вороновича [1] связывает с алгеброй Хопфа $\mathcal{A}$ алгебру внешних форм $\Gamma$ и обертывающую алгебру $\mathcal{U}$ левоинвариантных векторных полей на $\mathcal{A}$. Алгебра $\mathcal{U}$ называется квантовой алгеброй Ли ( $q$-алгеброй Ли для краткости). Она определяется соотношениями

$$
\chi_{i} \chi_{j}-\sigma_{i j}^{k m} \chi_{k} \chi_{m}=C_{i j}^{k} \chi_{k}
$$

где $\left\{\chi_{i}\right\}$ - набор генераторов, а структурные константы $\sigma_{i j}^{k m}$ и $C_{i j}^{k}$ подчиняются определенным связям (см. раздел 2). q-Алгебра Ли является неоднородной квадратичной алгеброй. Общая теория квадратичных алгебр рассматривалась в ряде работ (см., например, [2]-[4]). Отметим, что случай $q$-алгебр Ли весьма специален. В работе [1] был построен аналог комплекса де Рама для алгебры $\mathcal{U}$. Следуя классической теории

\footnotetext{
* Department of Mathematics, University of Kentucky, USA. E-mail: vgorb@ms.uky.edu

$\dagger$ Лаборатория теоретической физики им. Н. Н. Боголюбова, Объединенный институт ядерных исследований, Дубна, Московская обл., Россия. E-mail: isaevap@thsun1.jinr.ru

${ }^{\ddagger}$ Center of Theoretical Physics, Luminy, Marseille, France; Физический институт им. П. Н. Лебедева РАН, Москва, Россия. Е-mail: oleg@cpt.univ-mrs.fr
} 
алгебр Ли, в статьях [5], [6] мы ввели квантовые аналоги стандартного комплекса и БРСТ-дифференциала с ожидаемыми свойствами (обзор общей БРСТ-теории можно найти в [7]). В данной работе мы продолжаем исследование $q$-алгебр Ли в духе теории алгебр Ли. А именно, стандартный комплекс $q$-алгебры Ли отображается в подпространство $q$-антисимметричных цепей bar-комплекса для $\mathcal{U}$ (см., например, [8]). Оказывается, что образ этого отображения в точности, как и в случае алгебр Ли, является подкомплексом bar-комплекса для $\mathcal{U}$. Более того, квантовый БРСТ-дифференциал является ограничением bar-дифференциала $b$ на подпространство $q$-антисимметричных цепей.

Работа построена следующим образом. В разделе 2 напоминается определение $q$-алгебр Ли. В разделе 3 вводятся построенные Вороновичем внешние расширения $q$-алгебр Ли (алгебра внешних форм Г и внутренних производных $\Gamma^{*}$; отметим, что наше определение базиса дифференциальных форм слегка отличается от определения Вороновича, см. [5]) и развивается аппарат, необходимый для построения основных конструкций в разделах 4 и 5 . Раздел 4 содержит явную конструкцию БРСТ-оператора $q$-алгебры Ли. Изложение в разделах 2-4 следует работе [9]. В разделе 5 обсуждаются bar-комплекс $\left(C_{n}(\mathcal{U}), b\right)$ для $q$-алгебры Ли, его подкомплекс $q$-антисимметричных цепей $\left(C_{n}(\mathcal{U}, \wedge \mathcal{L}), b\right)$ и цепное отображение стандартного комплекса для $q$-алгебры Ли в $\left(C_{n}(\mathcal{U}, \wedge \mathcal{L}), b\right)$. Для доказательства основных предложений в разделах 4 и 5 используются нетривиальные тождества в групповой алгебре группы кос. Раздел 6 посвяшен обобшению вышеизложенной конструкции на случай, когда $q$-алгебра Ли снабжена оператором градуировки. Примером может служить супералгебра Ли с градуировкой, задаваемой оператором четности. Известно, что имеются два выбора коммутационных соотношений между бозонными и фермионными духовыми переменными. Мы объясняем эквивалентность этих двух выборов в рамках теории $q$-алгебр Ли, снабженных оператором градуировки. В разделе 7 суммируются результаты данной работы.

\section{2. ОПРЕДЕЛЕНИЯ И ОБОЗНАЧЕНИЯ}

Данные, задаюшие квантовую алгебру Ли с $N$ генераторами, удобно закодировать в виде следующей $\left((N+1)^{2} \times(N+1)^{2}\right)$-матрицы [10]:

$$
\begin{aligned}
R_{A B}^{C D} & =\delta_{A}^{i} \delta_{B}^{j} \sigma_{i j}^{k l} \delta_{k}^{C} \delta_{l}^{D}+\delta_{A}^{i} \delta_{B}^{j} C_{i j}^{k} \delta_{0}^{C} \delta_{k}^{D}+\delta_{A}^{i} \delta_{i}^{D} \delta_{B}^{0} \delta_{0}^{C}+\delta_{B}^{C} \delta_{A}^{0} \delta_{0}^{D} \equiv \\
& \equiv \delta_{A}^{\langle 1} \delta_{B}^{\langle 2} \sigma_{12} \delta_{1\rangle}^{C} \delta_{2\rangle}^{D}+\delta_{A}^{\langle 1} \delta_{B}^{\langle 2} C_{12\rangle}^{\langle 2} \delta_{0}^{C} \delta_{2\rangle}^{D}+\delta_{A}^{\langle 1} \delta_{1\rangle}^{D} \delta_{B}^{0} \delta_{0}^{C}+\delta_{B}^{C} \delta_{A}^{0} \delta_{0}^{D}
\end{aligned}
$$

Здесь индексы $1,2, \ldots$ - номера $N$-мерных векторных пространств $V_{N} ;\langle 1,\langle 2, \ldots$ и 1$\rangle, 2\rangle, \ldots$ - соответственно исходящие и входящие векторы; строчные буквы $i, j, k, l, \ldots=1,2, \ldots, N$ обозначают индексы векторного пространства $V_{N} ;$ прописные буквы $A, B, \ldots=0,1, \ldots, N$ обозначают индексы $(N+1)$-мерного пространства $V_{N+1}$ $\left(V_{N}\right.$ является подпространством в $\left.V_{N+1}\right)$.

Уравнения (1) эквивалентны следующим условиям:

$$
R_{k l}^{i j}=\sigma_{k l}^{i j}, \quad R_{k l}^{0 j}=C_{k l}^{j}, \quad R_{B 0}^{0 A}=R_{0 B}^{A 0}=\delta_{B}^{A},
$$


причем остальные компоненты матрицы $R$ обрашаются в нуль. Мы предполагаем, что матрица $\sigma \in \operatorname{End}\left(V_{N} \otimes V_{N}\right)$ имеет собственное значение 1.

Пусть $L_{A}=\left\{\chi_{0}, \chi_{i}\right\}, i=1, \ldots, N,-$ "квантовый вектор"

$$
L_{A}=\delta_{A}^{\langle 1} \chi_{1\rangle}+\delta_{A}^{0} \chi_{0}=\left(\begin{array}{c}
\chi_{0} \\
\chi_{i}
\end{array}\right)
$$

для матрицы $R$ :

$$
R_{A B}^{C D} L_{C} L_{D}=L_{A} L_{B}
$$

Эти соотношения эквивалентны следуюшим:

$$
\left[\chi_{0}, \chi_{i}\right]=0, \quad\left(1-\sigma_{12}\right) \chi_{1\rangle} \chi_{2\rangle}=C_{12\rangle}^{\langle 1} \chi_{0} \chi_{1\rangle} .
$$

Можно выполнить масштабное преобразование $\chi_{i} \rightarrow \chi_{0} \chi_{i}$. Преобразованные таким образом генераторы $\chi_{i}, i=1,2, \ldots, N$, удовлетворяют соотношениям

$$
\left(1-\sigma_{12}\right) \chi_{1\rangle} \chi_{2\rangle}=C_{12\rangle}^{\langle 1} \chi_{1\rangle},
$$

а соответствуюшая алгебра называется квантовой алгеброй Лu, если матрица $R$ удовлетворяет уравнению Янга-Бакстера

$$
R_{A B}^{C D} R_{M C}^{E S} R_{S D}^{F N}=R_{M A}^{C S} R_{S B}^{D N} R_{C D}^{E F} \quad \text { или } R_{\underline{23}} R_{12} R_{\underline{23}}=R_{\underline{12}} R_{\underline{23}} R_{12}
$$

(здесь индексы $\underline{12}$ или $\underline{23}$ обозначают номера векторных пространств $V_{N+1}$, в которых $R$-матрица действует нетривиально). Уравнение Янга-Бакстера для $R$ накладывает следующие условия на структурные константы квантовой алгебры Ли (5):

$$
\begin{aligned}
\sigma_{1} \sigma_{2} \sigma_{1} & =\sigma_{2} \sigma_{1} \sigma_{2}, \\
C_{1} \delta_{3} C_{1} & =\sigma_{2} C_{1} \delta_{3} C_{1}+C_{2} C_{1}, \\
C_{1} \delta_{3} \sigma_{1} & =\sigma_{2} \sigma_{1} C_{2}, \\
\left(\sigma_{2} C_{1} \delta_{3}+C_{2}\right) \sigma_{1} & =\sigma_{1}\left(\sigma_{2} C_{1} \delta_{3}+C_{2}\right) .
\end{aligned}
$$

Мы использовали здесь сокрашенные обозначения $\sigma_{n}:=\sigma_{n, n+1}, C_{n}:=C_{n, n+1\rangle}^{\langle n}, \delta_{n}:=$ $\delta_{n\rangle}^{\langle n-1}$

\section{3. ВНЕШНИЕ РАСШИРЕНИЯ КВАНТОВОЙ АЛГЕБРЫ ЛИ}

Для построения внешнего расширения квантовой алгебры Ли (5) вводятся квантовые аналоги внешних алгебр с генераторами $\gamma_{i}$ и $\Omega^{i}, i=1, \ldots, N$ :

$$
\begin{gathered}
\Omega^{\langle n} \wedge \cdots \wedge \Omega^{\langle 2} \wedge \Omega^{\langle 1}=\Omega^{\langle n} \otimes \cdots \otimes \Omega^{\langle 2} \otimes \Omega^{\langle 1} A_{1 \rightarrow n} \\
\gamma_{1\rangle} \wedge \gamma_{2\rangle} \wedge \cdots \wedge \gamma_{n\rangle}=A_{1 \rightarrow n} \gamma_{1\rangle} \otimes \gamma_{2\rangle} \otimes \cdots \otimes \gamma_{n\rangle} .
\end{gathered}
$$


Перекрестные коммутационные соотношения имеют вид

$$
\gamma_{2\rangle} \Omega^{\langle 2}=-\Omega^{\langle 1} \sigma_{12}^{-1} \gamma_{1\rangle}+I_{2}
$$

где $I_{2}$ - единичная матрица, действующая во втором векторном пространстве. В уравнениях (11) и (12) используются операторы $A_{1 \rightarrow n}$ - квантовые аналоги антисимметризаторов. Они определяются по индукции (в действительности операторы $A_{1 \rightarrow n}$ имеют смысл уже для групповой алгебры группы кос $B_{M+1}$, если заменить матрищы $\sigma_{i}$ генераторами $\hat{\sigma}_{i}, i=1, \ldots, M$; см. замечание 1 ниже):

$$
A_{1 \rightarrow n} \equiv f_{1 \rightarrow n} A_{1 \rightarrow n-1} \text { или } A_{1 \rightarrow n} \equiv \bar{f}_{1 \rightarrow n} A_{2 \rightarrow n},
$$

где $A_{1 \rightarrow 1}=1, f_{k \rightarrow k}=\bar{f}_{k \rightarrow k}=1$ и

$$
\begin{aligned}
& f_{k \rightarrow m}=1-f_{k \rightarrow m-1} \sigma_{m-1} \quad \text { или } \quad f_{k \rightarrow m}=f_{k+1 \rightarrow m}+(-1)^{m-k} \sigma_{k} \ldots \sigma_{m-1}, \\
& \bar{f}_{k \rightarrow m}=1-\bar{f}_{k+1 \rightarrow m} \sigma_{k} \quad \text { или } \quad \bar{f}_{k \rightarrow m}=\bar{f}_{k \rightarrow m-1}+(-1)^{m-k} \sigma_{m-1} \ldots \sigma_{k+1} \sigma_{k}
\end{aligned}
$$

при $k<m \leqslant n$. В явном виде имеем

$$
\begin{aligned}
& f_{k \rightarrow n} \equiv f_{k \rightarrow n}^{(\sigma)}=1-\sigma_{n-1}+\sigma_{n-2} \sigma_{n-1}-\cdots+(-1)^{n-k} \sigma_{k} \sigma_{k+1} \ldots \sigma_{n-1} \\
& \bar{f}_{k \rightarrow n} \equiv \bar{f}_{k \rightarrow n}^{(\sigma)}=1-\sigma_{k}+\sigma_{k+1} \sigma_{k}-\cdots+(-1)^{n-k} \sigma_{n-1} \ldots \sigma_{k+1} \sigma_{k}
\end{aligned}
$$

Если последовательность операторов $A_{1 \rightarrow n}$ обрывается на шаге $n=h+1\left(A_{1 \rightarrow n}=0\right.$ $\forall n>h)$, то число $h$ называется высотой матрицы $\sigma_{12}=\rho\left(\hat{\sigma}_{12}\right)$.

Элементы $\gamma_{i}$ и $\Omega^{i}$ играют роль квантовых аналогов духовых переменных. Выбор знака и появление матрицы $\sigma_{12}^{-1}$ в правой части (13) соответствуют стандартному случаю (супер)алгебр Ли. Коммутационные соотношения $\chi_{i}$ с $\gamma_{j}$ и $\Omega^{k}$ имеют вид

$$
\chi_{2\rangle} \Omega^{\langle 2}=\Omega^{\langle 1}\left(\sigma_{12} \chi_{1\rangle}+C_{12\rangle}^{\langle 2}\right), \quad \gamma_{1\rangle} \chi_{2\rangle}=\sigma_{12} \chi_{1\rangle} \gamma_{2\rangle}+C_{12\rangle}^{\langle 1} \gamma_{1\rangle}
$$

Алгебра (5), (11)-(13) и (17) является внешним расширением квантовой алгебры Ли (см. [5]). Эта алгебра порождает дифференциальное исчисление Картана на квантовых группах в подходе Вороновича [1].

ЗАМЕЧАНИЕ 1 . Пусть $\hat{\sigma}_{i}, i=1, \ldots, M,-$ генераторы группы кос $B_{M+1}$ :

$$
\hat{\sigma}_{i} \hat{\sigma}_{i+1} \hat{\sigma}_{i}=\hat{\sigma}_{i+1} \hat{\sigma}_{i} \hat{\sigma}_{i+1}, \quad\left[\hat{\sigma}_{i}, \hat{\sigma}_{j}\right]=0 \text { при }|i-j|>1
$$

Важные свойства элементов $f_{k \rightarrow n}^{(\hat{\sigma})}$ и $\bar{f}_{k \rightarrow n}^{(\hat{\sigma})}(16)$ групповой алгебры $B_{M+1}$ состоят в том, чTO

$$
\begin{aligned}
& f_{1 \rightarrow n}^{(\hat{\sigma})} f_{1 \rightarrow n-1}^{(\hat{\sigma})} \ldots f_{1 \rightarrow m}^{(\hat{\sigma})}=x_{n}^{(n-m+1)} A_{m \rightarrow n} \\
& \bar{f}_{1 \rightarrow n}^{(\hat{\sigma})} \bar{f}_{2 \rightarrow n}^{(\hat{\sigma})} \ldots \bar{f}_{m \rightarrow n}^{(\hat{\sigma})}=x_{n}^{(n-m)} A_{1 \rightarrow m}
\end{aligned}
$$


для $n \geqslant m \geqslant 1$. Здесь $x_{n}^{(0)}=1, x_{m}^{(1)}=f_{1 \rightarrow m}^{(\hat{\sigma})}, x_{n}^{(n)}=1, x_{n}^{(n-1)}=\bar{f}_{1 \rightarrow n}^{(\hat{\sigma})}$ и $x_{m+1}^{(2)}$ имеет вид

$$
\begin{aligned}
x_{m+1}^{(2)}= & f_{1 \rightarrow m}^{(\hat{\sigma})}+f_{1 \rightarrow m-1}^{(\hat{\sigma})}\left(\hat{\sigma}_{m} \hat{\sigma}_{m-1}\right)+f_{1 \rightarrow m-2}^{(\hat{\sigma})}\left(\hat{\sigma}_{m-1} \hat{\sigma}_{m-2}\right)\left(\hat{\sigma}_{m} \hat{\sigma}_{m-1}\right)+\cdots \\
& \cdots+f_{1 \rightarrow 2}^{(\hat{\sigma})}\left(\hat{\sigma}_{3} \hat{\sigma}_{2}\right) \ldots\left(\hat{\sigma}_{m} \hat{\sigma}_{m-1}\right)+\left(\hat{\sigma}_{2} \hat{\sigma}_{1}\right) \ldots\left(\hat{\sigma}_{m} \hat{\sigma}_{m-1}\right) .
\end{aligned}
$$

Тождества (19), (20) эквивалентны формулам факторизации

$$
A_{1 \rightarrow n}=x_{n}^{(n-m)} A_{1 \rightarrow m} A_{m+1 \rightarrow n}
$$

Элементы $x_{n}^{(m)}$ можно определить индуктивно, используя рекуррентное соотношение

$$
x_{n+1}^{(n-m+1)}=x_{n}^{(n-m)}-(-1)^{n-m} x_{n}^{(n-m+1)} \hat{\sigma}_{n} \ldots \hat{\sigma}_{m} .
$$

В частности, имеем

$$
x_{n+1}^{(2)}=f_{1 \rightarrow n}^{(\hat{\sigma})}+x_{n}^{(2)} \hat{\sigma}_{n} \hat{\sigma}_{n-1}, \quad n \geqslant 2,
$$

что дает (21). Тогда имеем $x_{n+1}^{(3)}=x_{n}^{(2)}-x_{n}^{(3)} \hat{\sigma}_{n} \hat{\sigma}_{n-1} \hat{\sigma}_{n-2}$ при $n \geqslant 3$ и т.д.

Заметим, что элементы $x_{n}^{(m)}$ являются альтернированными суммами по элементам группы кос, которые можно рассматривать как квантовые аналоги $(m, n-m)$-перетасовок (перетасовки получаются проекцией $\hat{\sigma}_{i} \rightarrow-s_{i}$, где $s_{i}$ - генераторы симметрической группы $\left.S_{M+1}\right)$. С этой точки зрения внешние произведения $(11),(12)$ связаны с квантовыми перетасовочными (shuffle) произведениями (о квантовых перетасовках и соответствующих произведениях см. [11], [1]). Ассоциативность этих произведений гарантируется тождествами $x_{n}^{(n-m)} x_{m}^{(m-k)}=x_{n}^{(n-k)}\left(T_{k} x_{n-k}^{(n-m)} T_{k}^{-1}\right)$ при $k<m<n$, где $T_{k} \hat{\sigma}_{i} T_{k}^{-1}=\hat{\sigma}_{i+k}$.

\section{4. БРСТ-ОПЕРАТОР ДЛЯ КВАНТОВОЙ АЛГЕБРЫ ЛИ}

В работе [5] мы привели рекурсию, которая определяет БРСТ-оператор $Q$ для квантовой алгебры Ли.

ПРЕДЛОЖЕНИЕ 1. БРСТ-оператор $Q$ для квантовых алгебр Ли (5), удовлетворяющий уравнению $Q^{2}=0$, должсен иметь вид

$$
Q=\Omega^{i} \chi_{i}+\sum_{r=1}^{h-1} Q_{(r)}
$$

где $h$ - высота сплетающей матрицы $\sigma_{12}$, операторы $Q_{(r)}$ задаются выражениямu

$$
Q_{(r)}=\Omega^{\langle r+1|} \wedge \Omega^{\langle r|} \wedge \cdots \wedge \Omega^{\langle 1|} X_{|1 \ldots r+1\rangle}^{\langle 1 \ldots r|} \gamma_{|1\rangle} \wedge \cdots \wedge \gamma_{|r\rangle}
$$

$a X_{|1 \ldots r+1\rangle}^{\langle 1 \ldots r|}-$ тензоры, которые подчиняются рекуррентным соотношениям

$$
A_{1 \rightarrow r+1} X_{|1 \ldots r+1\rangle}^{\langle 1 \ldots r|} A_{1 \rightarrow r}=A_{1 \rightarrow r+1}\left((-1)^{r} \sigma_{r} \sigma_{r-1} \ldots \sigma_{1}-\mathbf{1}\right) X_{|2 \ldots r+1\rangle}^{\langle 2 \ldots r|} A_{2 \rightarrow r}
$$

2 Теоретическая и математическая физика, т. 139, № 1, 2004 г. 
с начальным условием $A_{12} X_{|12\rangle}^{\langle 1|}=-C_{|12\rangle}^{\langle 1|}$.

Следует подчеркнуть, что вывод рекурсии (26) требует рассмотрения лишь линейных по $\chi_{i}$ членов в уравнении $Q^{2}=0$.

В работе [9] было приведено решение уравнений (26) и явно определены коэффициенты $(A X A)_{|1 \ldots r+1\rangle}^{\langle 1 \ldots r|}$. При этом было использовано важное тождество в групповой алгебре групшы кос.

Лемма 1. Пусть $\hat{\sigma}_{i}, i=1, \ldots, M,-$ генераторы группы кос $B_{M+1}$ (18). Тогда виполнено следующее тохдество в групповой алгебре $B_{M+1}$ :

$$
Y_{1 \rightarrow r+1} \bar{f}_{1 \rightarrow r}^{(\hat{\sigma})}=\left((-1)^{r+1} f_{1 \rightarrow r+1}^{(\hat{\sigma})} \hat{\sigma}_{r} \ldots \hat{\sigma}_{1}+\bar{f}_{1 \rightarrow r+1}^{(\hat{\sigma})}\right) Y_{2 \rightarrow r+1}
$$

где $f_{1 \rightarrow r}^{(\hat{\sigma})} u \bar{f}_{1 \rightarrow r}^{(\hat{\sigma})}$ определяются в (16) $u$

$$
Y_{k \rightarrow r+1}=\left(1-\hat{\sigma}_{r}^{2}\right)\left(1+\hat{\sigma}_{r-1} \hat{\sigma}_{r}^{2}\right) \ldots\left(1-(-1)^{r-k} \hat{\sigma}_{k} \hat{\sigma}_{k+1} \ldots \hat{\sigma}_{r-1} \hat{\sigma}_{r}^{2}\right), \quad Y_{k \rightarrow k}=1 .
$$

ДоКАЗАТЕЛЬСТво. С помощью уравнений (15) можно представить тождество (27) в виде

$$
Y_{1 \rightarrow r+1} \bar{f}_{1 \rightarrow r}^{(\hat{\sigma})}=F_{1 \rightarrow r+1} Y_{2 \rightarrow r+1},
$$

где

$$
F_{1 \rightarrow r+1}=(-1)^{r} f_{1 \rightarrow r}^{(\hat{\sigma})} \hat{\sigma}_{r}^{2} \ldots \hat{\sigma}_{1}+\bar{f}_{1 \rightarrow r}^{(\hat{\sigma})} .
$$

Тождество (29) доказывается по индукции (см. [9], [12]).

ЗАмЕчАниЕ 2. Операторы $Y_{k \rightarrow r+1}(28)$ имеют другое представление:

$$
Y_{k \rightarrow r+1}=\left(1+\hat{\sigma}_{r}\right)\left(1-\hat{\sigma}_{r-1} \hat{\sigma}_{r}\right) \ldots\left(1+(-1)^{r-k} \hat{\sigma}_{k} \hat{\sigma}_{k+1} \ldots \hat{\sigma}_{r}\right) f_{k \rightarrow r+1}^{(\hat{\sigma})}
$$

Это представление есть не что иное, как квантовый вариант факторизационных тождеств Цагира [13] (которые можно получить из (28), (31) проекцией $\hat{\sigma}_{i} \rightarrow q s_{i}$, где $s_{i}-$ генераторы симметрической группы, $\left.s_{i}^{2}=1\right)$. Доказательство тождеств (31) проводится непосредственно (см., например, [9]).

Имеется следующий результат [9]:

ПРЕДЛОЖЕНИЕ 2. Явное решение рекуррентнъх соотношений (26) дается формулой

$$
\begin{aligned}
& (-1)^{r+1}\left(A_{1 \rightarrow r+1} X A_{1 \rightarrow r}\right)_{j_{1} \ldots j_{r+1}}^{i_{1} \ldots i_{r}}= \\
& =\left(\left(1-R_{\underline{r}}^{2}\right)\left(1+R_{\underline{r-1}} R_{\underline{r}}^{2}\right) \ldots\left(1+(-1)^{r} R_{\underline{1}} \ldots R_{\underline{r-1}} R_{\underline{r}}^{2}\right)\right)_{j_{1} \ldots j_{r} j_{r+1}}^{k_{1} \ldots k_{r} 0}\left(A_{1 \rightarrow r}\right)_{k_{1} \ldots k_{r}}^{i_{1} \ldots i_{r}} .
\end{aligned}
$$

Здесь $\underline{1}, \ldots, \underline{r-1}, \underline{r}$ нумеруют әкземплярь векторного пространства $V_{N+1} ; R_{\underline{r}}:=$ $R_{r, r+1} u \underline{r}, \underline{r+1}$ - номера векторных пространств $V_{N+1}$, в которых $R$-матрица (1) действует нетривиально; $i_{1}, \ldots, j_{1}, \ldots, k_{1}, \ldots=1,2, \ldots, N$ - векторные индексы. 
ДоКАЗАТЕЛЬСТвО. Используя определение антисимметризатора (14) и соотношения (7) в группе кос, перепишем правую часть (26) в виде

$$
\begin{aligned}
& A_{1 \rightarrow r+1}\left((-1)^{r} \sigma_{r} \sigma_{r-1} \ldots \sigma_{1}-1\right) X_{|2 \ldots r+1\rangle}^{\langle 2 \ldots r|} A_{2 \rightarrow r}= \\
& \quad=\left((-1)^{r} f_{1 \rightarrow r+1} \sigma_{r} \sigma_{r-1} \ldots \sigma_{1}-\bar{f}_{1 \rightarrow r+1}\right) A_{2 \rightarrow r+1} X_{|2 \ldots r+1\rangle}^{\langle 2 \ldots r|} A_{2 \rightarrow r} .
\end{aligned}
$$

Таким образом, выражение (32) является решением уравнения (26), если выполнено тождество

$$
\left(Y_{1 \rightarrow r+1}\right)_{1 \ldots r+1\rangle}^{\langle 1 \ldots r 0} \bar{f}_{1 \rightarrow r}=\left((-1)^{r+1} f_{1 \rightarrow r+1} \sigma_{r} \ldots \sigma_{1}+\bar{f}_{1 \rightarrow r+1}\right)\left(Y_{2 \rightarrow r+1}\right)_{2 \ldots r+1\rangle}^{\langle 2 \ldots r 0},
$$

где

$$
Y_{\underline{k \rightarrow r+1}}=\left(1-R_{\underline{r}}^{2}\right)\left(1+R_{\underline{r-1}} R_{\underline{r}}^{2}\right) \ldots\left(1+(-1)^{r} R_{\underline{k}} R_{\underline{k+1}} \ldots R_{\underline{r-1}} R_{\underline{r}}^{2}\right) .
$$

Согласно структуре индексов векторное пространство $V_{N+1}$ является прямой суммой $V_{N}$ и одномерного векторного пространства $V_{0}$. Пусть $P: V_{N+1} \rightarrow V_{N}$ и $1-P: V_{N+1} \rightarrow V_{0}-$ соответствующие проекторы. Аналогично пусть $\bar{P}$ и $1-\bar{P}-$ проекторы, связанные с дуальными пространствами $\bar{V}_{N+1}$.

Определим

$$
P_{1 \rightarrow r}:=P_{1} \ldots P_{r}, \quad P^{1 \rightarrow r 0}=\bar{P}_{1} \ldots \bar{P}_{r}(1-\bar{P})_{r+1} .
$$

Эти операторы разделяют компоненты, которые нам нужны.

Уравнение (33) можно теперь переписать в виде

$$
\begin{aligned}
& P_{1 \rightarrow r+1} Y_{\underline{1 \rightarrow r+1}} \bar{f}_{\underline{1 \rightarrow r}}^{(R)} P^{1 \rightarrow r 0}= \\
& \quad=P_{1 \rightarrow r+1}\left((-1)^{r+1} f_{\underline{1 \rightarrow r+1}}^{(R)} R_{\underline{r}} \ldots R_{\underline{1}}+\underline{\bar{f}_{1 \rightarrow r+1}^{(R)}}\right) \underline{Y_{\underline{2 \rightarrow r+1}}} P^{1 \rightarrow r 0}
\end{aligned}
$$

(где $f_{\underline{f_{\rightarrow n}}}^{(R)}$ и $\bar{f}_{\underline{1 \rightarrow n}}^{(R)}-(N+1)$-мерные аналоги операторов $\left.(16)\right)$ в силу соотношений

$$
\begin{aligned}
& \bar{f}_{\underline{1 \rightarrow r}}^{(R)} P^{1 \rightarrow r}=P^{1 \rightarrow r} \bar{f}_{1 \rightarrow r}^{(\sigma)}, \\
& Y_{2 \rightarrow r+1} P^{1 \rightarrow r 0}=P^{1 \rightarrow r+1}\left(P_{2 \rightarrow r+1} \underline{Y}_{2 \rightarrow r+1} P^{2 \rightarrow r 0}\right) \equiv \\
& \equiv P^{1 \rightarrow r+1}\left(\underline{Y}_{2 \rightarrow r+1}\right)_{2 \ldots r+1\rangle}^{\langle 2 \ldots r 0}, \\
& P_{1 \rightarrow r+1}\left((-1)^{r} f_{\underline{1 \rightarrow r+1}}^{(R)} R_{\underline{r}} \ldots R_{\underline{1}}-\bar{f}_{\underline{1 \rightarrow r+1}}^{(R)}\right) P^{1 \rightarrow r+1}= \\
& =\left((-1)^{r} f_{1 \rightarrow r+1} \sigma_{r} \sigma_{r-1} \ldots \sigma_{1}-\bar{f}_{1 \rightarrow r+1}\right) \text {, }
\end{aligned}
$$

которые можно получить непосредственно, используя явный вид $R$-матрицы (2).

Уравнение (34) выполнено в силу тождества (27), которое было доказано в лемме 1. Действительно, если в тождестве (27) взять $R$-матричное представление группы кос $\rho_{R}\left(B_{M+1}\right): \rho_{R}\left(\hat{\sigma}_{k}\right)=R_{\underline{k}}$ и подействовать на это тождество слева и справа проекторами $P_{1 \rightarrow r+1}, P^{1 \rightarrow r 0}$, то получим (34). Этим завершается доказательство предложения. 
В следуюшем разделе нам понадобятся дополнительные сведения о рассмотренном выше $R$-матричном представлении группы кос. Введем элементы Юциса-Мэрфи $J_{r} \in$ $\rho_{R}\left(B_{M+1}\right)$ :

$$
J_{1}=1, \quad J_{r+1}=R_{\underline{r}} J_{r} R_{\underline{r}} .
$$

Эти элементы образуют коммутируюший набор в $\rho_{R}\left(B_{M+1}\right),\left[J_{r}, J_{m}\right]=0$, и удовлетворяют равенствам

$$
R_{\underline{m}} J_{r}=J_{r} R_{\underline{m}}, \quad m<r .
$$

ПрЕДЛОЖЕНИЕ 3. Явный вид Компонент

$$
Z_{r+1}:=P_{1 \rightarrow r+1}\left(J_{r+1}\right) P^{1 \rightarrow r 0}
$$

әлементов Юииса-Мәрфи (35) в терминах структурных констант $C_{n}$ и $\sigma_{m} \partial а$ ется формулой

$$
Z_{r+1}=C_{r}+\sigma_{r} C_{r-1} \delta_{r+1}+\sigma_{r} \sigma_{r-1} C_{r-2} \delta_{r} \delta_{r+1}+\cdots+\sigma_{r} \sigma_{r-1} \ldots \sigma_{2} C_{1} \delta_{3} \ldots \delta_{r+1}
$$

Элементы $Z_{r}$ обладают свойствами

$$
\begin{gathered}
\sigma_{m} Z_{r}=Z_{r} \sigma_{m}, \quad m \leqslant r-2, \\
Z_{r+1}=C_{r}+\sigma_{r} Z_{r} \delta_{r+1} .
\end{gathered}
$$

ДокАЗАТЕЛЬСтво. Используя определение элементов Юциса-Мэрфи (35) и представление (2), можно переписать $P_{1 \rightarrow r+1}\left(J_{r+1}\right) P^{1 \rightarrow r 0}$ в виде

$$
P_{1 \rightarrow r+1}\left(R_{\underline{\underline{r}}} \ldots\left(R_{\underline{1}}\right)^{2} \ldots R_{\underline{r}}\right) P^{1 \rightarrow r 0}=P_{1 \rightarrow r+1}\left(R_{\underline{\underline{r}}} R_{\underline{r-1}} \ldots R_{\underline{1}}\right) P^{02 \rightarrow r+1} \delta_{2} \delta_{3} \ldots \delta_{r+1} .
$$

Теперь, снова используя (2), выводим (37). Для получения свойства (38) применим к (36) операторы $P_{1 \rightarrow r}$ и $P^{1 \rightarrow r-10}$ слева и справа (при $m \leqslant r-2$ ). Рекуррентное соотношение (39) является прямым следствием соотношений (37).

\section{5. СВЯЗЬ С ЦЕПНЫМ ВАR-КОМПЛЕКСОМ}

Напомним сначала некоторые стандартные факты. Для каждой ассоциативной алгебры $\mathcal{U}$ строится bar-комплекс $\left(C_{n}(\mathcal{U}), b\right)$ :

а) $C_{n}(\mathcal{U})$ является пространством $\underbrace{\mathcal{U} \otimes \cdots \otimes \mathcal{U}}_{n}$;

б) для цепи

$$
T=a_{1} \otimes a_{2} \otimes \cdots \otimes a_{n} \in C_{n}(\mathcal{U})
$$

действие граничного оператора $b: C_{n+1}(\mathcal{U}) \rightarrow C_{n}(\mathcal{U})$ имеет вид

$$
b\left(a_{1} \otimes a_{2} \otimes \cdots \otimes a_{n+1}\right)=\sum_{i=1}^{n}(-1)^{n-i}\left(a_{1} \otimes \cdots \otimes a_{i} a_{i+1} \otimes \cdots \otimes a_{n+1}\right) .
$$


Используя ассоциативность алгебры $\mathcal{U}$, можно непосредственно проверить, что $b^{2}\left(a_{1} \otimes a_{2} \otimes \cdots \otimes a_{n+1}\right)=0$. Более того, для алгебры $\mathcal{U}$ с единичным элементом комплекс $\left(C_{n}(\mathcal{U}), b\right)$ является ацикличным, поскольку можно определить оператор гомотопии $\delta$ : $C_{n}(\mathcal{U}) \rightarrow C_{n+1}(\mathcal{U})$,

$$
\delta\left(a_{1} \otimes a_{2} \otimes \cdots \otimes a_{n}\right)=\left(a_{1} \otimes a_{2} \otimes \cdots \otimes a_{n} \otimes 1\right),
$$

который удовлетворяет равенству $\delta b+b \delta=\mathrm{id}$.

Пусть $\mathcal{U}-q$-алгебра Ли с генераторами $\chi_{i}$ и определяюшими соотношениями (5). Рассмотрим цепи $T(40)$, где все элементы $a_{k}$ принадлежат набору $\left\{\chi_{j}\right\}$ генераторов $q$-алгебры Ли, и определим их $q$-антисимметричную линейную комбинацию, задаваемую сверткой с числовым тензором $A_{1 \rightarrow n} \in \operatorname{End}\left(V^{\otimes n}\right)(14)$ :

$$
\chi_{1\rangle} \wedge \chi_{2\rangle} \wedge \cdots \wedge \chi_{n\rangle}:=A_{1 \rightarrow n}\left\{\chi_{1\rangle} \otimes \chi_{2\rangle} \otimes \cdots \otimes \chi_{n\rangle}\right\} \in C_{n}(\mathcal{U}) .
$$

В действительности нам понадобятся цепи следующего вида:

$$
\left.a \otimes\left\{\chi_{1\rangle} \wedge \chi_{2}\right\rangle \wedge \cdots \wedge \chi_{n\rangle}\right\} \in C_{n+1}(\mathcal{U})
$$

с произвольным $a \in \mathcal{U}$. Обозначим подпространство таких цепей как $C_{n+1}(\mathcal{U}, \wedge \mathcal{L}) \in \mathcal{U} \otimes$ $\underbrace{\mathcal{L} \wedge \cdots \wedge \mathcal{L}}_{n}$, где $\mathcal{L}$ - линейная оболочка элементов $\left\{\chi_{i}\right\}$.

Сформулируем теперь один из основных результатов данной работы.

ПРЕДЛОЖЕНИЕ 4. Граничный оператор b (41) сохраняет q-антисимметрию,

$$
b: C_{n+1}(\mathcal{U}, \wedge \mathcal{L}) \longrightarrow C_{n}(\mathcal{U}, \wedge \mathcal{L})
$$

Другими словами, комплекс $\left(C_{n}(\mathcal{U}, \wedge \mathcal{L}), b\right)$ является подкомплексом bаг-комплекса $\left(C_{n}(\mathcal{U}), b\right)$.

ДокАЗАТЕльСтво. Действуя граничным оператором (41) на $q$-антисимметричные цепи (43), находим, что

$$
\begin{gathered}
b\left\{a \otimes \chi_{1\rangle} \wedge \chi_{2\rangle} \wedge \cdots \wedge \chi_{n+1\rangle}\right\}=(-1)^{n} A_{1 \rightarrow n+1}\left\{a \chi_{1\rangle} \otimes \chi_{2\rangle} \otimes \cdots \otimes \chi_{n+1\rangle}\right\}+ \\
+a \otimes A_{1 \rightarrow n+1}\left[\sum_{k=1}^{n}(-1)^{n-k}\left\{\chi_{1\rangle} \otimes \cdots \otimes \chi_{k\rangle} \chi_{k+1\rangle} \otimes \cdots \otimes \chi_{n+1\rangle}\right\}\right]
\end{gathered}
$$

или, преобразуя далее,

$$
\begin{aligned}
& b\left\{a \otimes \chi_{1\rangle} \wedge \chi_{2\rangle} \wedge \cdots \wedge \chi_{n+1\rangle}\right\}=(-1)^{n} \bar{f}_{1 \rightarrow n+1}\left\{a \chi_{1\rangle} \otimes \chi_{2\rangle} \wedge \cdots \wedge \chi_{n+1\rangle}\right\}+ \\
& \quad+a \otimes A_{1 \rightarrow n+1}\left[\sum_{k=1}^{n}(-1)^{n-k} t_{k, k+1\rangle}^{\langle k}\left\{\chi_{1\rangle} \otimes \cdots \otimes \chi_{k\rangle} \otimes \chi_{k+2\rangle} \otimes \cdots \otimes \chi_{n+1\rangle}\right\}\right],
\end{aligned}
$$


где структурные константы $t_{j k}^{i}$ удовлетворяют соотношениям

$$
C_{k, k+1\rangle}^{\langle k}=\left(1-\sigma_{k}\right) t_{k, k+1\rangle}^{\langle k},
$$

тензоры $\bar{f}_{1 \rightarrow n+1}$ определены согласно (14)-(16) и мы воспользовались определяющими соотношениями (5) и свойствами "антисимметризаторов"

$$
A_{1 \rightarrow n+1} \chi_{k\rangle} \chi_{k+1\rangle}=A_{1 \rightarrow n+1} t_{k, k+1\rangle}^{\langle k} \chi_{k\rangle}
$$

Таким образом, для доказательства отображения (44) надо представить второй член в (46) в виде

$$
a \otimes W_{1 \ldots n+1\rangle}^{\langle 1 \ldots n}\left\{\chi_{1\rangle} \wedge \cdots \wedge \chi_{n\rangle}\right\}=a \otimes W_{1 \ldots n+1\rangle}^{\langle 1 \ldots n} A_{1 \rightarrow n}\left\{\chi_{1\rangle} \otimes \cdots \otimes \chi_{n\rangle}\right\} .
$$

Поэтому достаточно доказать следующую лемму.

ЛЕмма 2. Существуют такие тензоры $W_{n+1}:=W_{1 \ldots n+1\rangle}^{\langle 1 \ldots n}$, что

$$
A_{1 \rightarrow n+1}\left(\sum_{k=1}^{n}(-1)^{n-k} t_{k, k+1\rangle}^{\langle k} \delta_{k+2 \rightarrow n+1}\right)=W_{n+1} A_{1 \rightarrow n}
$$

где $\delta_{n+1 \rightarrow n}:=1, \quad \delta_{k+2 \rightarrow n+1}:=\delta_{k+2} \ldots \delta_{n+1}-$ оператор сдвига $i \rightarrow i+1$ nрu $k<$ $i \leqslant n$. Явный вид тензоров $W_{n}$ (в терминах структурных констант $\sigma_{k l}^{i j}$ и $C_{j k}^{i}$ квантовой алгебры Ли) дается формулами

$$
\begin{aligned}
W_{n+1}= & C_{n}-\left(1-\sigma_{n}\right) C_{n-1} \delta_{n+1}+\left(1-\sigma_{n-1}+\sigma_{n} \sigma_{n-1}\right) C_{n-2} \delta_{n} \delta_{n+1}-\cdots \\
& \cdots+(-1)^{n}\left[1-\sigma_{2}+\sigma_{3} \sigma_{2}-\cdots+(-1)^{n+1} \sigma_{n} \ldots \sigma_{2}\right] C_{1} \delta_{3} \ldots \delta_{n+1}= \\
= & Z_{n+1}-Z_{n} \delta_{n+1}+Z_{n-1} \delta_{n} \delta_{n+1}+\cdots+(-1)^{n} Z_{2} \delta_{3} \ldots \delta_{n+1},
\end{aligned}
$$

где әлементы $Z_{k}$ определены в (37), (39) (мьи фиксируем $Z_{2}=C_{1}=-W_{2}$ ).

ДокАЗАТЕЛЬСтво. Чтобы доказать равенство (49), используем тождества (7)-(10) для структурных констант и соотношение $(47)$ между $C_{i k}^{j}$ и $t_{i k}^{j}$. Доказательство проведем по индукции.

Уравнение (49), очевидно, выполняется при $n=1$. Предположим, что (49) справедливо при $n=m-1$. Требуется доказать, что оно справедливо при $n=m$. В левой части (49) при $n=m$ получаем

$$
\begin{aligned}
A_{1 \rightarrow m+1} & \left(\sum_{k=1}^{m}(-1)^{m-k} t_{k, k+1\rangle}^{\langle k} \delta_{k+2} \ldots \delta_{m+1}\right)= \\
= & A_{1 \rightarrow m+1}\left(\left[\sum_{k=1}^{m-1}(-1)^{m-k} t_{k, k+1\rangle}^{\langle k} \delta_{k+2} \ldots \delta_{m}\right] \delta_{m+1}+t_{m, m+1\rangle}^{\langle m}\right)= \\
= & \left(-f_{m+1} W_{m} \delta_{m+1}+f_{m+1} f_{m} t_{m, m+1\rangle}^{\langle m}\right) A_{1 \rightarrow m-1}= \\
= & \left(-f_{m+1} W_{m} \delta_{m+1}+x_{m+1} C_{m}\right) A_{1 \rightarrow m-1} .
\end{aligned}
$$


Во втором равенстве мы использовали $(14),(49)$ при $n=m-1$; в третьем равенстве использовали (19) при $n-m=2$. Здесь и ниже мы полагаем $f_{m}:=f_{1 \rightarrow m}$ и $x_{m}:=x_{m}^{(2)}$ для упрощения обозначений.

Сравнивая полученное выражение с правой частью (49), мы можем записать уравнение

$$
-f_{m+1} W_{m} \delta_{m+1}+x_{m+1} C_{m}=W_{m+1} f_{m},
$$

которое является тождеством при $m=2$ в силу (9) и (10). Докажем (51) по индукции. Для этого заметим (см. (50)), что имеется рекуррентное соотношение

$$
W_{n+1}=Z_{n+1}-W_{n} \delta_{n+1} .
$$

Подставим в левую часть (51) рекуррентные соотношения $(15),(23),(52)$ и учтем соотношения $(9),(38),(39)$ и базу индукции:

$$
\begin{aligned}
-W_{m} & \delta_{m+1}+f_{m} \sigma_{m}\left(Z_{m}-W_{m-1} \delta_{m}\right) \delta_{m+1}+\left(f_{m}+x_{m} \sigma_{m} \sigma_{m-1}\right) C_{m}= \\
& =-W_{m} \delta_{m+1}+f_{m}\left(\sigma_{m} Z_{m} \delta_{m+1}+C_{m}\right)+W_{m} f_{m-1} \delta_{m+1} \sigma_{m-1}= \\
& =\left(Z_{m+1}-W_{m} \delta_{m+1}\right)-f_{m-1} \sigma_{m-1} Z_{m+1}+W_{m} \delta_{m+1} f_{m-1} \sigma_{m-1}= \\
& =\left(Z_{m+1}-W_{m} \delta_{m+1}\right)\left(1-f_{m-1} \sigma_{m-1}\right)=W_{m+1} f_{m} .
\end{aligned}
$$

Таким образом, доказано уравнение (51), а соответственно и (49). Лемма доказана.

Этим завершается доказательство предложения.

Тождество (49) гарантирует, что $q$-антисимметричные цепи (43) образуют подкомплекс в bar-комплексе $q$-алгебры Ли $\mathcal{U}(5)$. Тождество

$$
(b)^{2}\left\{a \otimes \chi_{1\rangle} \wedge \chi_{2\rangle} \wedge \cdots \wedge \chi_{n+1\rangle}\right\}=0
$$

для граничного оператора (45) непосредственно следует из тождества $b^{2}=0$ для barдифференциала $b$ (41).

По аналогии с теорией обычных алгебр Ли (см., например, [8]) мы свяжем комплекс $\left(C_{n}(\mathcal{U}, \wedge \mathcal{L}), b\right)$ со стандартным комплексом для квантовой алгебры Ли. А именно, мы соотнесем цепи

$$
C_{n}=a \otimes A_{1 \rightarrow n}\left(\chi_{1\rangle} \otimes \cdots \otimes \chi_{n}\right) \in C_{n}(\mathcal{U}, \wedge \mathcal{L})
$$

с полиномами внешних произведений $\gamma$ с коэффициентами в $\mathcal{U}$,

$$
M_{n}=a \gamma_{1\rangle} \wedge \gamma_{2\rangle} \wedge \cdots \wedge \gamma_{n\rangle}, \quad a \in \mathcal{U},
$$

с помошью взаимно однозначного отображения i: $C_{n} \leftrightarrow M_{n}$,

$$
\mathbf{i}\left(a\left\{\gamma_{1\rangle} \wedge \gamma_{2\rangle} \wedge \cdots \wedge \gamma_{n\rangle}\right\}\right)=a \otimes A_{1 \rightarrow n}\left\{\chi_{1\rangle} \otimes \chi_{2\rangle} \otimes \cdots \otimes \chi_{n\rangle}\right\} .
$$

БРСТ-оператор $Q(24),(25),(32)$ действует на выражения (53) справа, и согласно (13) переменные $\Omega^{i}$ в $Q$ следует рассматривать как "правые $q$-производные" по $\gamma$ (в частности, $\forall a \in \mathcal{U}$ правое действие $\Omega^{i}$ на $a$ дает нуль). 
ПРЕДЛОЖенИЕ 5. Справедливы следующие утверждения:

1) $Q^{2}=0$

2) отображение $\mathbf{i}(54)$ является цепныц отображением, $b \circ \mathbf{i}=\mathbf{i} \circ Q$.

ДокАЗАтЕльСтво. Рассмотрим только первые два члена в выражении (24):

$$
Q=\Omega^{\langle 1} \chi_{1\rangle}-\Omega^{\langle 2} \wedge \Omega^{\langle 1} t_{12\rangle}^{\langle 1} \gamma_{1\rangle}+\cdots
$$

Используя (13), (17), (37) и (39), можно доказать по индукции следуюшие соотношения:

$$
\begin{aligned}
\gamma_{1\rangle} \wedge \cdots \wedge \gamma_{n\rangle} \Omega^{\langle n}= & (-1)^{n} \Omega^{\langle 0} \sigma_{0}^{-1} \ldots \sigma_{n-1}^{-1} \gamma_{0\rangle} \wedge \cdots \wedge \gamma_{n-1\rangle}+ \\
& +(-1)^{n-1} \bar{f}_{1 \rightarrow n}^{(\sigma)} \sigma_{1}^{-1} \ldots \sigma_{n-1}^{-1} \gamma_{1\rangle} \wedge \cdots \wedge \gamma_{n-1\rangle}, \\
\gamma_{1\rangle} \wedge \cdots \wedge \gamma_{n-1\rangle} \chi_{n\rangle}= & \sigma_{n-1} \ldots \sigma_{1} \chi_{1\rangle} \gamma_{2\rangle} \wedge \cdots \wedge \gamma_{n\rangle}+Z_{n} \gamma_{1\rangle} \wedge \cdots \wedge \gamma_{n-1\rangle} .
\end{aligned}
$$

Таким образом, с использованием (20) и (56) правое действие на (53) первых двух членов (55) дает

$$
\begin{aligned}
Q\left(a \gamma_{1\rangle}\right. & \left.\wedge \cdots \wedge \gamma_{n\rangle}\right)=(-1)^{n-1} \bar{f}_{1 \rightarrow n}^{(\sigma)}\left(a \chi_{1\rangle} \gamma_{2\rangle} \wedge \cdots \wedge \gamma_{n\rangle}\right)+ \\
& +(-1)^{n-1} \bar{f}_{1 \rightarrow n}^{(\sigma)} \sigma_{1}^{-1} \ldots \sigma_{n-1}^{-1} Z_{n}\left(a \gamma_{1\rangle} \wedge \cdots \wedge \gamma_{n-1\rangle}\right)- \\
& -x_{n}^{(n-2)}\left(\sigma_{1}^{-1} \ldots \sigma_{n-1}^{-1}\right)\left(\sigma_{1}^{-1} \ldots \sigma_{n-2}^{-1}\right) C_{n-1}\left(a \gamma_{1\rangle} \wedge \cdots \wedge \gamma_{n-1\rangle}\right)+\cdots
\end{aligned}
$$

Согласно предложению 1 первые два члена в (55) совместно с условием

$$
\left.Q^{2}\right|_{\text {линейные по } \chi \text { члены }}=0
$$

определяют БРСТ-оператор $Q$ единственным образом. Поэтому необходимо только проверить, что формула (57) (при $n=1,2$ ) совместна с $b \circ \mathbf{i}=\mathbf{i} \circ Q$ и с уравнениями (46), (48), (54). Это можно сделать непосредственно. Поэтому $Q^{2}=0$, а і является цепным отображением.

Тот факт, что отображение i (54) является цепным, демонстрирует соотношение между стандартным комплексом для $q$-алгебры Ли (с нашим БРСТ-оператором) и подкомплексом $\left(C_{n}(\mathcal{U}, \wedge \mathcal{L}), b\right)$ bar-комплекса $\left(C_{n}(\mathcal{U}), b\right)$.

ЗАмечАниЕ 3 . Можно показать, что

$$
W_{n+1}=P_{1 \rightarrow n+1}\left(\bar{f}_{\underline{1 \rightarrow n+1}}^{(R)}-1\right) R_{\underline{1}} \ldots R_{\underline{n}} P^{1 \rightarrow n 0} .
$$

Соотношение (51) получается из тождества

$$
f_{\underline{1 \rightarrow m+1}}^{(R)}\left(\underline{f_{1 \rightarrow m}^{(R)}} R_{1} \ldots R_{\underline{m-1}}\right) R_{\underline{m}}=\left(\underline{f_{1 \rightarrow m+1}^{(R)}} R_{1} \ldots R_{\underline{m}}\right) f_{\underline{1 \rightarrow m}}^{(R)}
$$

действием на него операторами $P^{1 \rightarrow m, 0}$ и $P_{1 \rightarrow m+1}$ справа и слева. Тождество (59) эквивалентно тождеству

$$
\underline{f_{1 \rightarrow m+1}^{(R)}} \underline{\bar{f}_{1 \rightarrow m}^{(R)}}=\underline{\bar{f}_{1 \rightarrow m+1}^{(R)}} \underline{f_{2 \rightarrow m+1}^{(R)}},
$$


которое есть не что иное, как условие ассоциативности $x_{m+1}^{(1)} x_{m}^{(m-1)}=x_{m+1}^{(m)} T_{1} x_{m}^{(1)} T_{1}^{-1}$, рассмотренное в замечании 1. Для доказательства всех этих утверждений требуются соотношения

$$
\begin{gathered}
\left(\bar{f}_{\underline{1 \rightarrow n+1}}^{(R)}-1\right) R_{\underline{1}} \ldots R_{\underline{n}} P^{1 \rightarrow n 0}= \\
=P^{1 \rightarrow n+1} W_{n+1}+\sum_{k=1}^{n}(-1)^{k} P^{1 \rightarrow k}\left(1-P^{k+1}\right) P^{k+2 \rightarrow n+1} \delta_{k+2} \ldots \delta_{n+1}, \\
f_{\underline{1 \rightarrow m}}^{(R)} P^{1 \rightarrow m}=P^{1 \rightarrow m} f_{1 \rightarrow m}^{(\sigma)} .
\end{gathered}
$$

\section{6. ОБОБЩЕНИЕ $q$-АЛЕГБРЫ ЛИ}

6.1. Рассмотрим преобразование подобия $R$-матрицы

$$
R_{\underline{12}} \rightarrow R_{\underline{12}}^{\prime}=\mathcal{D}_{\underline{1}} \mathcal{D}_{\underline{2}} R_{\underline{12}} \mathcal{D}_{\underline{1}}^{-1} \mathcal{D}_{\underline{2}}^{-1},
$$

которое соответствует линейному преобразованию генераторов $L_{B} \rightarrow \mathcal{D}_{B}^{C} L_{C}$. Новая матрица (60) удовлетворяет уравнению Янга-Бакстера (6) для всех числовых матриц $\mathcal{D}$. Если матрица $\mathcal{D}$ имеет специальный вид

$$
\mathcal{D}_{0}^{0}=1, \quad \mathcal{D}_{i}^{0}=\mathcal{D}_{0}^{i}=0, \quad \mathcal{D}_{j}^{i}=D_{j}^{i},
$$

то новая $R$-матрица (60) имеет тот же вид (1) с преобразованными структурными константами:

$$
\sigma_{12} \rightarrow \sigma_{12}^{\prime}=D_{1} D_{2} \sigma_{12} D_{1}^{-1} D_{2}^{-1}, \quad C_{|12\rangle}^{\langle 2|} \rightarrow C_{|12\rangle}^{\prime\langle 2|}=D_{1} D_{2} C_{|12\rangle}^{\langle 2|} D_{2}^{-1} .
$$

Это преобразование оставляет инвариантным векторное пространство (обозначаемое через $\mathcal{L}$ ), линейно порожденное $N$ элементами $\chi_{i} \cdot q$-Алгебра Ли остается квадратичной при любом выборе базиса в $\mathcal{L}$, а структурные константы (5) изменяются согласно (61).

Интересный специальный случай возникает, ког да матрица $R$ сохраняется под действием преобразования (60), $R_{\underline{12}}=R_{\underline{12}}^{\prime}$. Тогда $(N \times N)$-матрица $D_{j}^{i}$ такова, что

$$
D_{1} D_{2} \sigma_{12}=\sigma_{12} D_{1} D_{2}, \quad D_{1} D_{2} C_{|12\rangle}^{\langle 2|}=C_{|12\rangle}^{\langle 2|} D_{2} \text {. }
$$

Лемма 3. Пусть $R_{\underline{12}}^{\prime}=R_{\underline{12}}$. Тогда $R$-матрича $\mathcal{D}_{\underline{1}} R_{\underline{12}} \mathcal{D}_{\underline{1}}^{-1}$ удовлетворяет уравнению Янга-Бакстера.

Доказательство проводится непосредственно.

Теперь можно рассмотреть квадратичную алгебру с определяюшими соотношениями

$$
\left(\mathcal{D}_{\underline{1}} R_{\underline{12}} \mathcal{D}_{\underline{1}}^{-1}\right) L_{\underline{1}\rangle}^{\prime} L_{\underline{2}\rangle}^{\prime}=L_{\underline{1}\rangle}^{\prime} L_{\underline{2}\rangle}^{\prime} \text {. }
$$

В компонентах для квантового вектора $L_{A}^{\prime}=\left\{\xi_{0}, \xi_{i}\right\}$ получаем

$$
\xi_{i} \xi_{0}=D_{i}^{j} \xi_{0} \xi_{j}, \quad\left(1-D_{1} \sigma_{12} D_{1}^{-1}\right) \xi_{1\rangle} \xi_{2\rangle}=D_{1} C_{12\rangle}^{\langle 1} \xi_{0} \xi_{1\rangle}
$$

Эта алгебра связана с q-алгеброй Ли (5) преобразованием

$$
\chi_{i}=\xi_{0}^{-1} \xi_{i} .
$$


6.2. Имеется обобщение внешней алгебры (11)-(13) и (17), возникаюшее в соответствии с только что проведенным рассмотрением. Можно ввести оператор градуировки $g$ с коммутационными соотношениями

$$
g \gamma_{1\rangle}=D_{1} \gamma_{1\rangle} g, \quad g \Omega^{\langle 1}=\Omega^{\langle 1} D_{1}^{-1} g, \quad g \chi_{1\rangle}=D_{1} \chi_{1\rangle} g
$$

где $D_{j}^{i}$ - матрица, удовлетворяющая (62). Заметим, что оператор градуировки $g$ можно “включить в игру", если рассмотреть квадратичную алгебру (63), положить (64) и взять $g=\xi_{0}^{-1}$.

Соотношения (65) согласованы с соотношениями (5), (11)-(13) и (17). Тогда дифференциальная алгебра Вороновича (11)-(13), (17) переписывается для новых генераторов

$$
\widetilde{\Omega}^{\langle 1}=\Omega^{\langle 1} g^{-1}, \quad \tilde{\gamma}_{1\rangle}=g \gamma_{1\rangle}
$$

в виде

$$
\begin{gathered}
\tilde{\gamma}_{2\rangle} \widetilde{\Omega}^{\langle 2}=-\widetilde{\Omega}^{\langle 1} D_{1}^{-1} \sigma_{12}^{-1} D_{1} \tilde{\gamma}_{1\rangle}+I_{2}, \\
\widetilde{\Omega}^{\langle 1} \wedge \widetilde{\Omega}^{\langle 2} \wedge \cdots \wedge \widetilde{\Omega}^{\langle n}=\widetilde{\Omega}^{\langle 1} \otimes \widetilde{\Omega}^{\langle 2} \otimes \cdots \otimes \widetilde{\Omega}^{\langle n} A_{1 \rightarrow n}^{(D)}, \\
\tilde{\gamma}_{1\rangle} \wedge \tilde{\gamma}_{2\rangle} \wedge \cdots \wedge \tilde{\gamma}_{n\rangle}=A_{1 \rightarrow n}^{(D)} \tilde{\gamma}_{1\rangle} \otimes \tilde{\gamma}_{2\rangle} \otimes \cdots \otimes \tilde{\gamma}_{n\rangle}, \\
\chi_{2\rangle} \widetilde{\Omega}^{\langle 2}=\widetilde{\Omega}^{\langle 1}\left(\sigma_{12} D_{1} \chi_{1\rangle}+C_{12\rangle}^{\langle 2}\right), \quad \tilde{\gamma}_{1\rangle} \chi_{2\rangle}=\sigma_{12} D_{1} \chi_{1\rangle} \tilde{\gamma}_{2\rangle}+C_{12\rangle}^{\langle 1} \tilde{\gamma}_{|1\rangle},
\end{gathered}
$$

где $A_{1 \rightarrow n}^{(D)}=D_{1}^{-1} \ldots D_{n-1}^{-1} A_{1 \rightarrow n} D_{1} \ldots D_{n-1}$.

Эта обобшенная алгебра вкладывается в bar-комплекс, причем вложение подобно тому, которое рассматривалось для частного случая $D=1$ (подробности мы опустим).

6.3. Квантовая алгебра Ли, определяемая равенствами (5), (7)-(10), обобщает обычные (супер)алгебры Ли. Действительно, в недеформированном случае сплетающая матрица $\sigma_{i j}^{m k}=(-1)^{(m)(k)} \delta_{j}^{m} \delta_{i}^{k}$ является матрицей суперперестановки (здесь $(m)=0,1-$ четность генератора $\chi_{m}$ ). Уравнение (7) выполняется тождественно (и, более того, мы имеем $\sigma^{2}=1$ ). Уравнение (8) совпадает с тождеством Якоби для (супер)алгебр Ли. Уравнение (9) тогда эквивалентно условию $\mathbb{Z}_{2}$-градуировки: $C_{j k}^{i}=0$ для $(i) \neq(j)+(k)$. В этом случае уравнение (10) следует из (9).

В этом смысле внешние алгебры (11)-(13) и (66)-(68) обобшают алгебры Гейзенберга фермионных (и бозонных) духовых и антидуховых переменных. Матрица градуировки $D$ при этом является матрицей $D_{n}^{m}=(-1)^{(n)} \delta_{n}^{m}$, которая определяет хорошо известньй автоморфизм супералгебр, и мы имеем

$$
\left(D_{1} \sigma_{12} D_{1}^{-1}\right)_{i j}^{m k}=-(-1)^{((m)+1)((k)+1)} \delta_{j}^{m} \delta_{i}^{k}
$$




\section{7. ЗАКЛЮЧЕНИЕ}

В данной работе мы исследовали специальный класс квадратичных алгебр $\mathcal{U}$ - квантовых алгебр Ли (5). Мы рассмотрели внешнее расширение алгебры $\mathcal{U}$, добавляя алгебру духовых переменных (11)-(13), (17), и нашли БРСТ-оператор $Q$. Используя БРСТоператор $(24),(25),(32)$, можно построить аналоги стандартного комплекса и комплекса де Рама для квантовых алгебр Ли. В данной работе показано, что имеется отображение $\mathbf{i}$, которое действует из стандартного комплекса в подкомплекс bar-комплекса для $\mathcal{U}$. Отображение і индуцирует (из bar-дифференциала $b$ ) дифференциал на стандартном комплексе. Мы сравнили этот индуцированный дифференциал с оператором $Q$. Оператор $Q$ единственным образом определяется двумя свойствами: начальными членами (55) и условием (58). Мы проверили, что индуцированный дифференциал удовлетворяет этим двум свойствам. Поэтому он совпадает с $Q$. Более того, отсюда следует, что $Q^{2}=0$. Для построения отображения $\mathbf{i}$ мы должны проверить некоторый набор нетривиальных тождеств на структурные константы $\sigma_{i j}^{k l}$ и $C_{i j}^{k}$. Подобные тождества появлялись и в индуктивном определении БРСТ-оператора для $q$-алгебр Ли. Изящное доказательство некоторых из этих тождеств основано на комбинировании констант $\sigma_{i j}^{k l}$ и $C_{i j}^{k}$ в бо́льшую матрицу $R_{C D}^{A B}$, реализуюшую $R$-матричное представление группы кос $\mathcal{B}_{\text {. }}$ Мы далее пользовались свойствами элементов Юциса-Мэрфив $\mathcal{B}$, квантовыми перетасовочными произведениями и несколькими важными тождествами в групповой алгебре группы кос.

Мы рассмотрели обобщение вышеизложенной конструкции на случай $q$-алгебры Ли, снабженной оператором градуировки.

Следует отметить, что ряд результатов из раздела 5 можно рассматривать как явную реализацию некоторых фактов из работы [3] для случая специального выбора неоднородных квадратичных алгебр.

Заметим, что частный пример нашей конструкции БРСТ-оператора для квантовой алгебры Ли $U_{q}(g l(N))$ уже был детально рассмотрен в нашей работе [14]. В этом случае мы также построили квантовые аналоги анти-БРСТ-оператора и квантового оператора Лапласа.

Мы надеемся, что наша конструкция БРСТ-оператора для квантовых алгебр Ли будет полезна для построения БРСТ-операторов в случае любых квадратичных алгебр (в том числе для бесконечномерных алгебр, таких как $W_{3}$ ), а также для конструирования квантовых $W$-алгебр из квантовых аффинных алгебр (см. [15] и приведенную там библиографию) посредством квантового аналога процедуры гамильтоновой редукции с помошью квантового БРСТ-метода.

Благодарности. Авторы признательны А. Герасимову, П. Этингофу и А. Полишуку за полезные обсуждения. В. Г. Горбунов и А. П. Исаев благодарят также Математический институт Макса Планка в Бонне и Марсельский университет, где была выполнена существенная часть данной работы, за гостеприимство и поддержку. Работа А. П. Исаева поддержана грантами РФФИ № 03-01-00781 и INTAS № 03-51-3350. Мы также благодарны рецензенту за полезные замечания. 


\section{Список литературы}

[1] S. L. Woronowicz. Commun. Math. Phys. 1989. V. 122. P. 125.

[2] B. L. Feigin, B. L. Tsygan. Cyclic homology of algebras with quadratic relations, universal enveloping algebras and group algebras. In: K-Theory, Arithmetic and Geometry. Lect. Notes in Math. V. 1289. Ed. Yu. I. Manin. New York: Springer, 1987. P. 210; P. Feng, B. L. Tsygan. Commun. Math. Phys. 1991. V. 140. P. 481.

[3] A. Braverman, D. Gaitsgory. J. Algebra. 1996. V. 181. P. 315; hep-th/9411113.

[4] A. Polishchuk, L. Positselski. Quadratic algebras. 1996 (неопубликовано).

[5] А. П. Исаев, О. В. Огиевецкий. ТМФ. 2001. Т. 129. № 2. C. 298; math.QA/0106206.

[6] Ч. Бурдик, А. П. Исаев, О. В. Огиевецкий. ЯФ. 2001. Т. 64. № 12. С. 2191; math.QA/0010060.

[7] M. Henneaux, C. Teitelboim. Quantization of Gauge Systems. Princeton: Princeton Univ. Press, 1992.

[8] А. Картан, С. Эйленберг. Гомологическая алгебра. М.: ИЛ, 1960.

[9] A. P. Isaev, O. Ogievetsky. BRST operator for quantum Lie algebras: explicit formula. Int. J. Mod. Phys. A. 2004 (in press).

[10] D. Bernard. Phys. Lett. B. 1991. V. 260. P. 389.

[11] M. Rosso. Invent. Math. 1998. V. 133. P. 133.

[12] M. Graña. Contemp. Math. 2000. V. 267. P. 111.

[13] D. B. Zagier. Commun. Math. Phys. 1992. V. 147. P. 199.

[14] A. P. Isaev, O. Ogievetsky. Nucl. Phys. B. 2001. V. 102. P. 306.

[15] A. Sevostyanov. Selecta Math. (N. S.). 2002. V. 8. P. 637; math.QA/0107215. 